\title{
Underserved, Rural Health
}

National Cancer Institute

\section{Source}

National Cancer Institute. Underserved, Rural Health. NCI Thesaurus. Code C19503.

This includes rural, elderly, low-literacy, blue collar, and poor populations. Minorities per se are not included but often belong to one or more or the included categories. 